\title{
Existence of Multiple Positive Periodic Solutions to Two Species Parasitical Model with Impulsive Effects and Harvesting Terms
}

\author{
Zhouhong Li \\ Department of Mathematics, Yuxi Normal University, Yuxi, Yunnan 653100, China \\ Correspondence should be addressed to Zhouhong Li; zhouhli@yeah.net
}

Received 15 July 2012; Revised 10 January 2013; Accepted 6 February 2013

Academic Editor: Eric R. Kaufmann

Copyright (C) 2013 Zhouhong Li. This is an open access article distributed under the Creative Commons Attribution License, which permits unrestricted use, distribution, and reproduction in any medium, provided the original work is properly cited.

By applying Mawhin's continuation theorem of coincidence degree theory and some skills of inequalities, we establish the existence of four positive solutions for two species parasitical system with impulsive effects and harvesting terms. Finally, an example is given to illustrate the effectiveness of our results.

\section{Introduction}

In recent years, the existence of periodic solutions in biological models has been widely studied. Models with harvesting terms are often considered. Generally, the model with harvesting terms is described as follows:

$$
\dot{x}_{1}=x_{1} f\left(x_{1}, x_{2}\right)-h, \quad \dot{x}_{2}=x_{2} g\left(x_{1}, x_{2}\right)-k,
$$

where $x_{1}$ and $x_{2}$ are functions of two species, respectively; $h$ and $k$ are harvesting terms standing for the harvests (see $[1,2])$. Because of the effect of changing environment such as the weather, season, and food, the number of species population periodically varies with the time. The rate of change usually is not a constant. Motivated by this, we consider the periodic nonautonomous population models. For example, two species parasitical system with harvesting terms is as follows [3]:

$$
\begin{gathered}
\dot{x}=x(t)\left(a_{1}(t)-b_{1}(t) x(t)\right)-h_{1}(t), \\
\dot{y}=y(t)\left(a_{2}(t)-b_{2}(t) y(t)+c(t) x(t)\right)-h_{2}(t),
\end{gathered}
$$

where $x(t)$ and $y(t)$ denote the densities of the host and the parasites, respectively; $a_{i}(t), b_{i}(t), c(t)$, and $h_{i}(t)(i=1,2)$ are all positive continuous functions and denote the intrinsic growth rate, death rate, obtaining nutriment rate from the host, and harvesting rate, respectively. In the model (2), the parasitical influence on its host is negligible. As we know, in population dynamics, many evolutionary processes experience short-time rapid chance after undergoing relatively long sooth variation. Examples include stocking of species and annual immigration. Incorporating these phenomena gives us impulsive differential equations. For the theory of impulsive differential equations, we refer the reader to $[4,5]$.

However, to the best of our knowledge, there are few results on the existence of multiple periodic solutions for the delay parasitical with impulsive effects in the literatures. This motivates us to consider the existence of multiple periodic solutions for following parasitical with impulsive effects and harvesting terms nonautonomous model:

$$
\begin{aligned}
& \dot{x}_{1}(t)= x_{1}(t)\left(a_{1}(t)-b_{1}(t) x_{1}\left(t-\tau_{11}(t)\right)\right) \\
&-h_{1}(t), \quad t \neq t_{k}, \\
& \dot{x}_{2}(t)= x_{2}(t)\left(a_{2}(t)-b_{2}(t) x_{2}\left(t-\tau_{22}(t)\right)\right. \\
&\left.+c(t) x_{1}\left(t-\tau_{21}(t)\right)\right)-h_{2}(t), \quad t \neq t_{k}, \\
& \Delta x_{i}\left(t_{k}\right)= x_{i}\left(t_{k}^{+}\right)-x_{i}\left(t_{k}^{-}\right)=g_{i k} x_{i}\left(t_{k}\right), \quad t=t_{k},
\end{aligned}
$$

where $g_{i k} \in(-1,+\infty)(i=1,2 ; k \in \mathbb{N}=\{1,2\})$. $\left\{t_{k}\right\}_{k \in \mathbb{N}}$ is a strictly increasing sequence with $t_{1}>0$ and $\lim _{t \rightarrow \infty} t_{k}=\infty \cdot x_{i}(t)(i=1,2)$ is the $i$ th species population density. $a_{i}(t)>0(i=1,2)$ denotes the intrinsic growth rate; $b_{i}(t)>0$ and $h_{i}(t)>0(i=1,2)$ stand for death rate, obtaining nutriment rate from the host, and harvesting rate, respectively. $c(t)>0$ represents obtaining nutriment rate from the host; $\tau_{21}(t) \geq 0$ stands for the time-lag in the process of transformation from the 1 th species to the 2 th species. 
$\tau_{i i}(t) \geq 0(i=1,2)$ represents the time-lag in the process of intraspecific competition.

In addition, the effects of a periodically varying environment are important for evolutionary theory as the selective forces on systems in a fluctuating environment differ from those in a stable environment. Therefore, the assumptions of periodicity of the parameters are a way of incorporating the periodicity of the environment (e.g, seasonal effects of weather, food supplies, mating habits, etc.), which leads us to assume that $a_{i}(t), b_{i}(t), c(t), \tau_{i i}(t), \tau_{21}(t)$, and $h_{i}(t)(i=$ $1,2)$ are all continuous $\omega$-periodic functions. For impulsive effects, we further assume that there exists a $q \in \mathbb{N}$ such that $g_{i(k+q)}=g_{i k}(i=1,2)$ and $t_{k+q}=t_{k}+\omega$.

Since a very basic and important problem in the study of a population growth model with a periodic environment is the global existence and stability of a positive periodic solution, which plays a similar role as a globally stable equilibrium does in an autonomous model, also, on the existence of positive periodic solutions to system (3), few results are found in the literatures. This motivates us to investigate the existence of a positive periodic or multiple positive periodic solutions for system (3). In fact, it is more likely for some biological species to take on multiple periodic change regulations and have multiple local stable periodic phenomena. Therefore, it is essential for us to investigate the existence of multiple positive periodic solutions for population models. Our main aim of this paper is by using Mawhin's continuation theorem of coincidence degree theory to establish the existence of four positive periodic solutions for system (3). For the work concerning the multiple existence of periodic solutions of periodic population models which was done using coincidence degree theory, we refer the reader to [6-8].

This paper is organized as follows. In Section 2, by using the continuation theorem of coincidence degree theory and the skills of inequalities, we establish the existence of at least four positive periodic solutions of system (3). An example is presented in the last section to illustrate the effectiveness of our results.

\section{Existence of at Least Four Positive Periodic Solutions}

We first summarize a few concepts from the book by Gaines and Mawhin [9].

Let $X$ and $Z$ be real normed vector spaces. Let $L$ : Dom $L \subset X \rightarrow Z$ be a linear mapping and $N: X \times[0,1] \rightarrow$ $Z$ a continuous mapping. The mapping $L$ will be called a Fredholm mapping of index zero if $\operatorname{dim} \operatorname{Ker} L=\operatorname{codim} \operatorname{Im} L<$ $\infty$, and $\operatorname{Im} L$ is closed in $Z$. If $L$ is a Fredholm mapping of index zero, then there exist continuous projectors $P: X \rightarrow$ $X$ and $Q: Z \rightarrow Z$ such that $\operatorname{Im} P=\operatorname{Ker} L$ and $\operatorname{Ker} Q=$ $\operatorname{Im} L=\operatorname{Im}(I-Q)$, and $X=\operatorname{Ker} L \oplus \operatorname{Ker} P, Z=\operatorname{Im} L \oplus \operatorname{Im} Q$. It follows that $\left.L\right|_{\text {Dom } L \cap K e r} P:(I-P) X \rightarrow \operatorname{Im} L$ is invertible, and its inverse is denoted by $K_{P}$. If $\Omega$ is a bounded open subset of $X$, the mapping $N$ is called $L$-compact on $\bar{\Omega} \times[0,1]$, and if $Q N(\bar{\Omega} \times[0,1])$ is bounded, $K_{P}(I-Q) N: \bar{\Omega} \times[0,1] \rightarrow X$ is compact. Because $\operatorname{Im} Q$ is isomorphic to $\operatorname{Ker} L$, there exists an isomorphism $J: \operatorname{Im} Q \rightarrow \operatorname{Ker} L$.
Lemma 1 (see [9]). Let $L$ be a Fredholm mapping of index zero, and let $N$ be L-compact on $\bar{\Omega} \times[0,1]$. Assume

(a) for each $\lambda \in(0,1)$, every solution $x$ of $L x=\lambda N(x, \lambda)$ is such that $x \notin \partial \Omega \cap \operatorname{Dom} L$;

(b) $Q N(x, 0) x \neq 0$ for each $x \in \partial \Omega \cap \operatorname{Ker} L$;

(c) $\operatorname{deg}(J Q N(x, 0), \Omega \cap \operatorname{Ker} L, 0) \neq 0$.

Then $L x=N(x, 1)$ has at least one solution in $\bar{\Omega} \cap \operatorname{Dom} L$.

Let $T$ be a given positive constant, and a finite number of points of the sequence $\left\{\tau_{k}\right\}$ lies in the interval $[0, T]$. Let $P C\left([0, T], \mathbb{R}^{n}\right)$ be the set of functions $x:[0, T] \rightarrow \mathbb{R}^{n}$ which are piecewise continuous in $[0, T]$ and have points of discontinuous $\tau_{k} \in[0, T]$, where they are continuous from the left. In the set $P C\left([0, T], \mathbb{R}^{n}\right)$ introduce the norm $\|x\|=$ $\sup |x(t)|: t \in[0, T]$ with which $P C\left([0, T], \mathbb{R}^{n}\right)$ becomes a Banach space with the uniform convergence topology.

Definition 2. The set $\mathscr{F} \subset P C\left([0, T], \mathbb{R}^{n}\right)$ is said to be quasiequicontinuous in $[0, T]$, if for any $\epsilon>0$, there exists $\delta>0$ such that if $x \in \mathscr{F} ; k \in \mathbb{Z} ; t_{1}, t_{2} \in\left(\tau_{k-1}, \tau_{k}\right] \cap[0, T]$ and $\left|t_{1}-t_{2}\right|<\delta$, then $\left|x\left(t_{1}\right)-x\left(t_{2}\right)\right|<\epsilon$.

The following result called compactness criterion gives a necessary and sufficient condition for relative compactness in $P C\left([0, T], \mathbb{R}^{n}\right)$.

Lemma 3 (see [4]). The set $\mathscr{F} \subset P C\left([0, T], \mathbb{R}^{n}\right)$ is relatively compact if and only if

(a) $\mathscr{F}$ is bounded, namely, $\|x\| \leq c$, for each $x \in \mathscr{F}$ and some $c>0$

(b) $\mathscr{F}$ is quasiequicontinuous in $[0, T]$.

For the sake of convenience, we denote $\bar{f}=(1 / \omega)$ $\int_{0}^{\omega} f(t) \mathrm{d} t$; here $f(t)$ is a continuous $\omega$-periodic function. Let $P C_{\omega}$ denote the space of $\omega$-periodic functions $\psi: \mathbb{R} \rightarrow \mathbb{R}$ which are continuous for $t \neq t_{k}$, are continuous from the left for $t \in \mathbb{R}$, and have possible discontinuities of the first kind at points $t=t_{k}$; that is, the limit from the right of $t_{\mathrm{k}}$ exists but may be different from the value at $t_{k}$. We also denote $P C_{\omega}^{1}=\left\{\psi \in P C_{\omega}: \dot{\psi} \in P C_{\omega}\right\}$. Obviously, $\psi$ is continuous if $\psi \in P C_{\omega}^{1}$.

For simplicity, we need to introduce some notations as follows:

$$
\begin{gathered}
g_{i}=\frac{1}{2} \sum_{k=1}^{q} \ln \left(1+g_{i k}\right), \\
G_{i}=\frac{1}{2} \sum_{k=1}^{q}\left[\ln \left(1+g_{i k}\right)+\left|\ln \left(1+g_{i k}\right)\right|\right] \\
\rho_{i}=e^{\bar{a}_{i} \omega+G_{i}}, \\
l_{1}^{ \pm}=\frac{\left(\bar{a}_{1}+2 g_{1} / \omega\right) \rho_{1} \pm \sqrt{\left[\left(\bar{a}_{1}+2 g_{1} / \omega\right) \rho_{1}\right]^{2}-4 \bar{b}_{1} \bar{h}_{1} \rho_{1}}}{2 \bar{b}_{1}},
\end{gathered}
$$




$$
\begin{gathered}
l_{2}^{ \pm}=\left(\left(\bar{c} l_{1}^{+}+a_{2}+\frac{2 g_{2}}{\omega}\right) \rho_{2}\right. \\
\pm \sqrt{\left.\left[\left(\bar{c} l_{1}^{+}+a_{2}+\frac{2 g_{2}}{\omega}\right) \rho_{2}\right]^{2}-4 \bar{b}_{2} \bar{h}_{2} \rho_{2}\right)}\left(2 \bar{b}_{2}\right)^{-1}, \\
A_{i}^{ \pm}=\frac{\left(\bar{a}_{i}+2 g_{i} / \omega\right) \pm \sqrt{\left(\bar{a}_{i}+2 g_{i} / \omega\right)^{2}-4 \bar{b}_{i} \bar{h}_{i} \rho_{i}}}{2 \bar{b}_{i}}, \\
B_{i}^{ \pm}=\frac{A_{i}^{ \pm}}{\rho_{i}}, \quad H_{i}=\frac{l_{i}^{-}}{\rho_{i}},
\end{gathered}
$$

where $i=1,2$. tions:

Throughout this paper, we need the following assump-

$$
\begin{aligned}
& \left(T_{1}\right)\left(\bar{a}_{i}+2 g_{i} / \omega\right) \rho_{i}^{-1 / 2}>2 \sqrt{\bar{b}_{i} \bar{h}_{i}}(i=1,2) ; \\
& \left(T_{2}\right) \rho_{i}>1(i=1,2) .
\end{aligned}
$$

The following results will play an important role in the proof of our main result.

Lemma 4 (see [5]). Suppose $\psi \in P C_{\omega}^{1}$, then

$$
\begin{aligned}
0 & \leq \sup _{s \in[0, \omega]} \psi(s)-\inf _{s \in[0, \omega]} \psi(s) \\
& \leq \frac{1}{2}\left[\int_{0}^{\omega}\left|\psi^{\prime}(s)\right| d s+\sum_{k=1}^{q}\left|\Delta \psi\left(t_{k}\right)\right|\right] .
\end{aligned}
$$

Lemma 5. Let $x>0, y>0, z>0$, and $x>2 \sqrt{y z}$; for the functions $f(x, y, z)=\left(x+\sqrt{x^{2}-4 y z}\right) / 2 z$ and $g(x, y, z)=$ $\left(x-\sqrt{x^{2}-4 y z}\right) / 2 z$, the following assertions hold.

(1) $f(x, y, z)$ and $g(x, y, z)$ are monotonically increasing and monotonically decreasing on the variable $x \in$ $(0, \infty)$, respectively.

(2) $f(x, y, z)$ and $g(x, y, z)$ are monotonically decreasing and monotonically increasing on the variable $y \in$ $(0, \infty)$, respectively.

(3) $f(x, y, z)$ and $g(x, y, z)$ are monotonically decreasing and monotonically increasing on the variable $z \in$ $(0, \infty)$, respectively.

Proof. In fact, for all $x>0, y>0$, and $z>0$, we have

$$
\begin{gathered}
\frac{\partial f}{\partial x}=\frac{x+\sqrt{x^{2}-4 y z}}{2 z \sqrt{x^{2}-4 y z}}>0, \\
\frac{\partial g}{\partial x}=\frac{\sqrt{x^{2}-4 y z}-x}{2 z \sqrt{x^{2}-4 y z}}<0, \\
\frac{\partial f}{\partial y}=\frac{-1}{\sqrt{x^{2}-4 y z}}<0,
\end{gathered}
$$

$$
\begin{gathered}
\frac{\partial g}{\partial y}=\frac{1}{\sqrt{x^{2}-4 y z}}>0, \\
\frac{\partial f}{\partial z}=\frac{-\left(x+\sqrt{x^{2}-4 y z}\right)^{2}}{4 z^{2} \sqrt{x^{2}-4 y z}}<0, \\
\frac{\partial g}{\partial z}=\frac{\left(x-\sqrt{x^{2}-4 y z}\right)^{2}}{4 z^{2} \sqrt{x^{2}-4 y z}}>0 .
\end{gathered}
$$

By the relationship of the derivative and the monotonicity, the above assertions obviously hold. The proof of Lemma 5 is complete.

Lemma 6. Assume that $\left(T_{1}\right)$ and $\left(T_{2}\right)$ hold, then one has the following inequalities:

$$
\begin{aligned}
& \text { (1) } \ln l_{i}^{+}>\ln A_{i}^{+}>\ln A_{i}^{-}>\ln l_{i}^{-}>\ln H_{i}(i=1,2) ; \\
& \text { (2) } \ln l_{i}^{+}>\ln B_{i}^{+}>\ln B_{i}^{-}>\ln l_{i}^{-}>\ln H_{i}(i=1,2) ; \\
& \text { (3) } \min \left\{\ln A_{i}^{+}, \ln B_{i}^{+}\right\}>\max \left\{\ln A_{i}^{-}, \ln B_{i}^{-}\right\}(i=1,2) .
\end{aligned}
$$

Proof. Since

$$
\begin{aligned}
\left(\bar{a}_{1}+\frac{2 g_{1}}{\omega}\right) \rho_{1} & >\bar{a}_{1}+\frac{2 g_{1}}{\omega} \\
& >\frac{\bar{a}_{1}+2 g_{1} / \omega}{\sqrt{\rho_{1}}}>2 \sqrt{\bar{b}_{1} \bar{h}_{1}}>0, \\
\left(\bar{c} l_{2}^{+}+2 a_{2}+\frac{2 g_{2}}{\omega}\right) \rho_{2}> & \bar{a}_{2}+\frac{2 g_{2}}{\omega} \\
> & \frac{2 g_{2} / \omega+\bar{a}_{2}}{\sqrt{\rho_{2}}}>2 \sqrt{\bar{b}_{2} \bar{h}_{2}}>0, \\
& \bar{b}_{i}>0, \bar{h}_{i}>0, i=1,2 .
\end{aligned}
$$

Applying Lemma 5, we have

$$
\begin{aligned}
0 & <H_{1}=\frac{l_{1}^{-}}{\rho_{1}}<l_{1}^{-}=g\left(\left(\bar{a}_{1}+\frac{2 g_{1}}{\omega}\right) \rho_{1}, \bar{b}_{1}, \bar{h}_{1} \rho_{1}\right) \\
& <g\left(\bar{a}_{1}+\frac{2 g_{1}}{\omega}, \bar{b}_{1}, \bar{h}_{1} \rho_{1}\right)=A_{1}^{-} \\
& <A_{1}^{+}=f\left(\bar{a}_{1}+\frac{2 g_{1}}{\omega}, \bar{b}_{1}, \bar{h}_{1} \rho_{1}\right) \\
& <f\left(\left(\bar{a}_{1}+\frac{2 g_{1}}{\omega}\right) \rho_{1}, \bar{b}_{1}, \bar{h}_{1} \rho_{1}\right)=l_{1}^{+}, \\
0 & <H_{2}=\frac{l_{2}^{-}}{\sqrt{\rho_{2}}}<l_{2}^{-}=g\left(\left(\bar{c}_{2}^{+}+\bar{a}_{2}+\frac{2 g_{2}}{\omega}\right) \rho_{2}, \bar{b}_{2}, \bar{h}_{2} \rho_{2}\right)
\end{aligned}
$$




$$
\begin{aligned}
& <g\left(\frac{2 g_{2}}{\omega}+\bar{a}_{2}, \bar{b}_{2}, \bar{h}_{2} \rho_{2}\right)=A_{2}^{-} \\
& <A_{2}^{+}=f\left(\frac{2 g_{2}}{\omega}+\bar{a}_{2}, \bar{b}_{2}, \bar{h}_{2} \rho_{2}\right) \\
& <f\left(\left(\bar{c} l_{1}^{+}+\bar{a}_{2}+\frac{2 g_{2}}{\omega}\right) \rho_{2}, \bar{b}_{2}, \bar{h}_{2} \rho_{2}\right)=l_{2}^{+} .
\end{aligned}
$$

Thus, we have $\ln H_{i}<\ln l_{i}^{-}<\ln A_{i}^{-}<\ln A_{i}^{+}<\ln l_{i}^{+}, \quad i=$ 1,2 . The proof of (1) of Lemma 6 is complete. For (2) of Lemma 6, we similarly have

$$
\begin{aligned}
0 & <H_{1}=\frac{l_{1}^{-}}{\rho_{1}}<l_{1}^{-}=g\left(\left(\bar{a}_{1}+\frac{2 g_{1}}{\omega}\right) \rho_{1}, \bar{b}_{1}, \bar{h}_{1} \rho_{1}\right) \\
& <g\left(\bar{a}_{1}+\frac{2 g_{1}}{\omega}, \bar{b}_{1}, \bar{h}_{1} \rho_{1}\right)=B_{1}^{-} \\
& <B_{1}^{+}=f\left(\bar{a}_{1}+\frac{2 g_{1}}{\omega}, \bar{b}_{1}, \bar{h}_{1} \rho_{1}\right) \\
& <f\left(\left(\bar{a}_{1}+\frac{2 g_{1}}{\omega}\right) \rho_{1}, \bar{b}_{1}, \bar{h}_{1} \rho_{1}\right)=l_{1}^{+}, \\
0 & <H_{2}=\frac{l_{2}^{-}}{\rho_{2}}<l_{2}^{-}=g\left(\left(\bar{a}_{2}+\frac{2 g_{2}}{\omega}\right) \rho_{2}, \bar{b}_{2}, \bar{h}_{2} \rho_{2}\right) \\
& <g\left(\left(\frac{2 g_{2}}{\omega}+\bar{a}_{2}\right) \rho_{2}, \bar{b}_{2}, \frac{\bar{h}_{2}}{\rho_{2}}\right)=B_{2}^{-}<B_{2}^{+} \\
& =f\left(\left(\frac{2 g_{2}}{\omega}+\bar{a}_{2}\right) \rho_{2}, \bar{b}_{2}, \frac{\bar{h}_{2}}{\rho_{2}}\right) \\
& <f\left(\left(\bar{c}_{1}^{+}+\bar{a}_{2}+\frac{2 g_{2}}{\omega}\right) \rho_{2}, \bar{b}_{2}, \bar{h}_{2} \rho_{2}\right)=l_{2}^{+},
\end{aligned}
$$

which imply that $\ln H_{i}<\ln l_{i}^{-}<\ln B_{i}^{-}<\ln B_{i}^{+}<\ln l_{i}^{+}(i=$ $1,2)$; that is, the inequality (2) of Lemma 6 holds. Let us now prove the inequality (3) of Lemma 6 . In fact, since

$$
\begin{aligned}
\max & \left\{\ln A_{i}^{-}, \ln B_{i}^{-}\right\} \\
& =\frac{\ln A_{i}^{-}+\ln B_{i}^{-}+\left|\ln A_{i}^{-}-\ln B_{i}^{-}\right|}{2} \quad(i=1,2),
\end{aligned}
$$$$
\min \left\{\ln A_{i}^{+}, \ln B_{i}^{+}\right\}
$$

$$
=\frac{\ln A_{i}^{+}+\ln B_{i}^{+}+\left|\ln A_{i}^{+}-\ln B_{i}^{+}\right|}{2} \quad(i=1,2),
$$

we have

$$
\begin{aligned}
\min & \left\{\ln A_{i}^{+}, \ln B_{i}^{+}\right\}-\max \left\{\ln A_{i}^{-}, \ln B_{i}^{-}\right\} \\
= & \left(\ln A_{i}^{+}+\ln B_{i}^{+}-\ln A_{i}^{-}-\ln B_{i}^{-}\right. \\
& \left.\quad-\left|\ln A_{i}^{-}-\ln B_{i}^{-}\right|-\left|\ln A_{i}^{+}-\ln B_{i}^{+}\right|\right)(2)^{-1} .
\end{aligned}
$$

If $A_{i}^{+} \geq B_{i}^{+}$, then $A_{i}^{-} \leq B_{i}^{-}$, and

$$
\begin{gathered}
\min \left\{\ln A_{i}^{+}, \ln B_{i}^{+}\right\}-\max \left\{\ln A_{i}^{-}, \ln B_{i}^{-}\right\} \\
=\ln B_{i}^{+}-\ln B_{i}^{-}>0 .
\end{gathered}
$$

$$
\begin{aligned}
& \text { If } A_{i}^{+}<B_{i}^{+} \text {, then } A_{i}^{-}>B_{i}^{-} \text {, and } \\
& \qquad \begin{aligned}
\min & \left\{\ln A_{i}^{+}, \ln B_{i}^{+}\right\}-\max \left\{\ln A_{i}^{-}, \ln B_{i}^{-}\right\} \\
& =\ln A_{i}^{+}-\ln A_{i}^{-}>0 .
\end{aligned}
\end{aligned}
$$

Hence, we drive $\min \left\{\ln A_{i}^{+}, \ln B_{i}^{+}\right\}>\max \left\{\ln A_{i}^{-}, \ln B_{i}^{-}\right\}$. From the above, we have completed the proof of Lemma 6 .

Theorem 7. Assume that $\left(T_{1}\right)$ and $\left(T_{2}\right)$ hold. Then system (3) has at least four positive $\omega$-periodic solutions.

Proof. By making the substitution

$$
x_{i}(t)=e^{u_{i}(t)}, \quad i=1,2
$$

system (3) can be reformulated as

$$
\begin{aligned}
\dot{u}_{1}(t)= & a_{1}(t)-b_{1}(t) e^{u_{1}\left(t-\tau_{11}(t)\right)} \\
& -h_{1}(t) e^{-u_{1}(t)}, \quad t \neq t_{k}, \\
\dot{u}_{2}(t)= & a_{2}(t)-b_{2}(t) e^{u_{2}\left(t-\tau_{22}(t)\right)} \\
& +c(t) e^{u_{1}\left(t-\tau_{21}(t)\right)}-h_{2}(t) e^{-u_{2}(t)}, \quad t \neq t_{k}, \\
\Delta u_{i}\left(t_{k}\right)= & u_{i}\left(t_{k}^{+}\right)-u_{i}\left(t_{k}^{-}\right) \\
= & \ln \left(1+g_{i k}\right), \quad i=1,2, \quad t=t_{k} . \\
\text { Let } \quad & \\
X=\{u=( & \left.\left(u_{1}, u_{2}\right)^{T}: u_{i} \in P C_{\omega}^{1}, \quad i=1,2\right\}, \quad Z=X \times \mathbb{R}^{2 q},
\end{aligned}
$$

and define

$$
\|u\|_{0}=\sum_{i=1}^{2} \sup _{t \in[0, \omega]}\left|u_{i}(t)\right|, \quad u \in X,
$$$$
\|z\|_{1}=\|u\|_{0}+\sum_{j=1}^{q}\left|\xi_{j}\right|, \quad z=\left(u, \xi_{1}, \xi_{2}, \ldots, \xi_{q}\right) \in Z .
$$

Then both $\left(X,\|\cdot\|_{0}\right)$ and $\left(Z,\|\cdot\|_{1}\right)$ are Banach spaces. Let

$\operatorname{Dom} L=\{u \in X: \dot{x} \in X\}, L: \operatorname{Dom} L \longrightarrow Z$,

$$
\begin{aligned}
L(u & \left.=\left(u_{1}, u_{2}\right)^{T}\right) \\
& =\left(\left(\begin{array}{l}
\dot{u}_{1} \\
\dot{u}_{2}
\end{array}\right),\left\{\left(\begin{array}{c}
\Delta u_{1}\left(t_{k}\right) \\
\Delta u_{2}\left(t_{k}\right)
\end{array}\right)\right\}_{k=1}^{q}\right),
\end{aligned}
$$

$N: X \longrightarrow Z$,

$$
N(u, \lambda)=\left(\left(\begin{array}{l}
F_{1}(t, \lambda) \\
F_{2}(t, \lambda)
\end{array}\right),\left\{\left(\begin{array}{l}
\ln \left(1+g_{1 k}\right) \\
\ln \left(1+g_{2 k}\right)
\end{array}\right)\right\}_{k=1}^{q}\right),
$$


where

$$
\begin{gathered}
F_{1}(t, \lambda)=a_{1}(t)-b_{1}(t) e^{u_{1}\left(t-\tau_{11}(t)\right)}-h_{1}(t) e^{-u_{1}(t)}, \\
F_{2}(t, \lambda)=a_{2}(t)-b_{2}(t) e^{u_{2}\left(t-\tau_{22}(t)\right)} \\
+\lambda c(t) e^{u_{1}\left(t-\tau_{21}(t)\right)}-h_{2}(t) e^{-u_{2}(t)}, \\
P: X \rightarrow X, \quad Q: Z \rightarrow Z, \\
P\left(u=\left(u_{1}, u_{2}\right)^{T}\right)=\left(\bar{u}_{1}, \bar{u}_{2}\right)^{T}, \quad u \in X, \\
Q\left(\left(\begin{array}{l}
u_{1} \\
u_{2}
\end{array}\right),\left\{\left(\begin{array}{l}
m_{1 k} \\
m_{2 k}
\end{array}\right)\right\}_{k=1}^{q}\right) \\
\left(\left(\begin{array}{l}
\bar{u}_{1}+\frac{1}{\omega} \sum_{k=1}^{q} m_{1 k} \\
\bar{u}_{2}+\frac{1}{\omega} \sum_{k=1}^{q} m_{2 k}
\end{array}\right),\left\{\left(\begin{array}{l}
0 \\
0
\end{array}\right)\right\}_{k=1}^{q}\right) .
\end{gathered}
$$

Obviously,

$$
\operatorname{Ker} L=\left\{u=\left(u_{1}, u_{2}\right)^{T} \in X:\right.
$$

$$
\left.\left(u_{1}(t), u_{2}(t)(t)\right)=\left(c_{1}, c_{2}\right) \in \mathbb{R}^{2}, \quad t \in \mathbb{R}\right\},
$$

$\operatorname{Im} L$

$$
=\left\{\left(\left(\begin{array}{l}
u_{1} \\
u_{2}
\end{array}\right),\left\{\left(\begin{array}{l}
m_{1 k} \\
m_{2 k}
\end{array}\right)\right\}_{k=1}^{q}\right) \in Z: \begin{array}{c}
\omega \bar{u}_{1}+\sum_{k=1}^{q} m_{1 k}=0 \\
\omega \bar{u}_{2}+\sum_{k=1}^{q} m_{2 k}=0
\end{array}\right\} \text {. }
$$

Since $\operatorname{Im} L$ is closed in $Z, \operatorname{Im} P=\operatorname{Ker} L, \operatorname{Ker} Q=\operatorname{Im} L$, and $\operatorname{dim} \operatorname{Ker} L=2=\operatorname{codim} \operatorname{Im} L$; we know that $L$ is a Fredholm mapping of index zero. Furthermore, the generalized inverse (to $L$ ) $K_{P}: \operatorname{Im} L \rightarrow \operatorname{Ker} P \bigcap \operatorname{Dom} L$ is given by

$$
\begin{aligned}
& K_{P}\left(z=\left(\left(\begin{array}{l}
u_{1} \\
u_{2}
\end{array}\right),\left\{\left(\begin{array}{l}
m_{1 k} \\
m_{2 k}
\end{array}\right)\right\}_{k=1}^{q}\right)\right) \\
& =\left(\begin{array}{l}
\int_{0}^{t} u_{1}(s) \mathrm{d} s+\sum_{0<t_{k}<t} m_{1 k}-\frac{1}{\omega} \sum_{k=1}^{q} m_{1 k}-\frac{1}{\omega} \int_{0}^{\omega} \int_{0}^{t} u_{1}(s) \mathrm{d} s \mathrm{~d} t \\
\int_{0}^{t} u_{2}(s) \mathrm{d} s+\sum_{0<t_{k}<t} m_{2 k}-\frac{1}{\omega} \sum_{k=1}^{q} m_{2 k}-\frac{1}{\omega} \int_{0}^{\omega} \int_{0}^{t} u_{2}(s) \mathrm{d} s \mathrm{~d} t
\end{array}\right) .
\end{aligned}
$$

Then

$$
\mathrm{QN}(u, \lambda)=\left(\left(\begin{array}{l}
\frac{1}{\omega} \int_{0}^{\omega} F_{1}(s, \lambda) \mathrm{d} s+\frac{2 g_{1}}{\omega} \\
\frac{1}{\omega} \int_{0}^{\omega} F_{2}(s, \lambda) \mathrm{d} s+\frac{2 g_{2}}{\omega}
\end{array}\right),\left\{\left(\begin{array}{l}
0 \\
0
\end{array}\right)\right\}_{k=1}^{q}\right),
$$

$$
\begin{aligned}
& K_{P}(I-Q) N(u, \lambda) \\
& =\left(\begin{array}{l}
\int_{0}^{t} F_{1}(s, \lambda) \mathrm{d} s+\sum_{0<t_{k}<t} \ln \left(1+g_{1 k}\right) \\
\int_{0}^{t} F_{1}(s, \lambda) \mathrm{d} s+\sum_{0<t_{k}<t} \ln \left(1+g_{1 k}\right)
\end{array}\right) \\
& +\left(\frac{1}{2}-\frac{t}{\omega}\right)\left(\begin{array}{l}
\int_{0}^{\omega} F_{1}(s, \lambda) \mathrm{d} s+2 g_{1} \\
\int_{0}^{\omega} F_{2}(s, \lambda) \mathrm{d} s+2 g_{2}
\end{array}\right) \\
& -\frac{1}{\omega}\left(\begin{array}{l}
\int_{0}^{\omega} \int_{0}^{t} F_{1}(s, \lambda) \mathrm{d} s \mathrm{~d} t+2 g_{1} \\
\int_{0}^{\omega} \int_{0}^{t} F_{2}(s, \lambda) \mathrm{d} s \mathrm{~d} t+2 g_{2}
\end{array}\right),
\end{aligned}
$$

where $F_{i}(s, \lambda)(i=1,2)$ are very similar to that of $F_{i}(t, \lambda)(i=$ $1,2)$. By the Lebesgue convergence theorem, $Q N$ and $K_{P}(I-$ Q) $N$ are continuous. Moreover, because of periodicity, it follows from Lemma 3 that $Q N(\bar{\Omega}), K_{P}(I-Q) N(\bar{\Omega})$ are relatively compact for any open bounded set $\Omega \subset X$. Thus, $N$ is $L$-compact on $\bar{\Omega}$ for any open bounded set $\Omega \subset X$.

In order to use Lemma 1, we have to find at least four appropriate open bounded subsets of $X$. Considering the operator equation $L u=\lambda N(u, \lambda), \lambda \in(0,1)$, we have

$$
\begin{gathered}
\dot{u}_{1}(t)=\lambda\left(a_{1}(t)-b_{1}(t) e^{u_{1}\left(t-\tau_{11}(t)\right)}-h_{1}(t) e^{-u_{1}(t)}\right), \\
\dot{u}_{2}(t)=\lambda\left(a_{2}(t)-b_{2}(t) e^{u_{2}\left(t-\tau_{22}(t)\right)}\right. \\
\left.+\lambda c(t) e^{u_{1}\left(t-\tau_{21}(t)\right)}-h_{2}(t) e^{-u_{2}(t)}\right), \\
\Delta u_{j}\left(t_{k}\right)=\lambda \ln \left(1+g_{j k}\right), \quad j=1,2 .
\end{gathered}
$$

Assume that $u \in X$ is a $\omega$-periodic solution of system (23) for some $\lambda \in(0,1)$. Integrating (23) from 0 to $\omega$, we have

$$
\begin{gathered}
-2 g_{1}=\int_{0}^{\omega}\left\{a_{1}(t)-b_{1}(t) e^{u_{1}\left(t-\tau_{11}(t)\right)}-h_{1}(t) e^{-u_{1}(t)}\right\} \mathrm{d} t, \\
-2 g_{2}=\int_{0}^{\omega}\left\{a_{2}(t)-b_{2}(t) e^{u_{2}\left(t-\tau_{22}(t)\right)}\right. \\
\left.+\lambda c(t) e^{u_{1}\left(t-\tau_{21}(t)\right)}-h_{2}(t) e^{-u_{2}(t)}\right\} \mathrm{d} t .
\end{gathered}
$$

Then

$$
\begin{aligned}
& \int_{0}^{\omega}\left\{b_{1}(t) e^{u_{1}\left(t-\tau_{11}(t)\right)}+h_{1}(t) e^{-u_{1}(t)}\right\} \mathrm{d} t=\bar{a}_{1} \omega+2 g_{1}, \\
& \int_{0}^{\omega}\left\{b_{2}(t) e^{u_{2}\left(t-\tau_{22}(t)\right)}-\lambda c(t) e^{u_{1}\left(t-\tau_{21}(t)\right)}+h_{2}(t) e^{-u_{2}(t)}\right\} \mathrm{d} t
\end{aligned}
$$

$$
=\bar{a}_{2} \omega+2 g_{2} \text {. }
$$

Furthermore, note that $u=\left(u_{1}, u_{2}\right)^{T} \in X$. There exists $\xi_{i}, \eta_{i} \in$ $[0, \omega], i=1,2$, such that $u_{i}\left(\xi_{i}\right)=\sup _{t \in[0, \omega]} u_{i}(t), u_{i}\left(\eta_{i}\right)=$ $\inf _{t \in[0, \omega]} u_{i}(t)$. 
On the one hand, according to the first equation of (23) and (25), we have

$$
\int_{0}^{\omega}\left|\dot{u}_{1}(t)\right| \mathrm{d} t<2 \bar{a}_{1} \omega+2 g_{1} .
$$

In the light of the first equation of (25) and (26) and Lemma 4, we get

$$
\begin{aligned}
& \omega \bar{b}_{1} e^{u_{1}\left(\xi_{1}\right)-\left(\bar{a}_{1} \omega+G_{1}\right)}+\omega \bar{h}_{1} e^{-u_{1}\left(\xi_{1}\right)} \\
& \quad \leq \omega \bar{b}_{1} e^{u_{1}\left(\xi_{1}\right)-(1 / 2)\left[\int_{0}^{\omega}\left|\dot{u}_{1}(s)\right| \mathrm{d} s+\sum_{k=1}^{q}\left|\Delta u_{1}\left(t_{k}\right)\right|\right]}+\omega \bar{h}_{1} e^{-u_{1}\left(\xi_{1}\right)} \\
& \quad<\omega \bar{b}_{1} e^{u_{1}\left(\eta_{1}\right)}+\omega \bar{h}_{1} e^{-u_{1}\left(\xi_{1}\right)} \\
& \quad \leq \int_{0}^{\omega}\left\{b_{1}(t) e^{u_{1}\left(t-\tau_{11}(t)\right)}+h_{1}(t) e^{-u_{1}(t)}\right\} \mathrm{d} t \\
& =\bar{a}_{1} \omega+2 g_{1},
\end{aligned}
$$

namely,

$$
\bar{b}_{1} e^{2 u_{1}\left(\xi_{1}\right)}-\left(\bar{a}_{1}+\frac{2 g_{1}}{\omega}\right) e^{\bar{a}_{1} \omega+G_{1}} e^{u_{1}\left(\xi_{1}\right)}+\bar{h}_{1} e^{\bar{a}_{1} \omega+G_{1}}<0,
$$

which implies that

$$
\ln l_{1}^{-}<u_{1}\left(\xi_{1}\right)<\ln l_{1}^{+} .
$$

Applying Lemma (23), we drive

$$
\ln H_{1}=\ln l_{1}^{-}-\left(\bar{a}_{1} \omega+G_{1}\right)<u_{1}(\eta) \leq u_{1}\left(\xi_{1}\right)<\ln l_{1}^{+} .
$$

According to (23) and (25), we get

$$
\int_{0}^{\omega}\left|\dot{u}_{2}(t)\right| \mathrm{d} t<2 \bar{a}_{2} \omega+2 g_{2} \text {. }
$$

By (25) and (31), we have

$$
\begin{aligned}
& \omega \bar{b}_{2} e^{u_{2}\left(\xi_{2}\right)-\left(\bar{a}_{2} \omega+G_{2}\right)}-\omega \bar{c} l_{1}^{+}+\omega \bar{h}_{2} e^{-u_{2}\left(\xi_{2}\right)} \\
& \leq \omega \bar{b}_{2} e^{u_{2}\left(\xi_{2}\right)-(1 / 2)\left[\int_{0}^{\omega}\left|\dot{u}_{2}(s)\right| \mathrm{d} s+\sum_{k=1}^{q}\left|\Delta u_{2}\left(t_{k}\right)\right|\right]} \\
&-\omega \bar{c} l_{1}^{+}+\omega \bar{h}_{2} e^{-u_{2}\left(\xi_{2}\right)} \\
&< \omega \bar{b}_{2} e^{u_{2}\left(\eta_{2}\right)}-\omega \bar{c} l_{1}^{+}+\omega \bar{h}_{2} e^{-u_{2}\left(\xi_{2}\right)} \\
&<\int_{0}^{\omega}\left\{b_{2}(t) e^{u_{2}\left(t-\tau_{22}(t)\right)}\right. \\
&\left.\quad-\lambda c(t) e^{u_{1}\left(t-\tau_{21}(t)\right)}+h_{2}(t) e^{-u_{2}(t)}\right\} \mathrm{d} t \\
&=\bar{a}_{2} \omega+2 g_{2},
\end{aligned}
$$

that is,

$$
\bar{b}_{2} e^{2 u_{2}\left(\xi_{2}\right)}-\left(\bar{c} l_{1}^{+}+a_{2}+\frac{2 g_{2}}{\omega}\right) e^{\bar{a}_{2} \omega+G_{2}} e^{u_{2}\left(\xi_{2}\right)}+\bar{h}_{2} e^{\bar{a}_{2} \omega+G_{2}}<0,
$$

which implies that

$$
\ln l_{2}^{-}<u_{2}\left(\xi_{2}\right)<\ln l_{2}^{+}
$$

Applying Lemma 4, we drive

$$
\ln H_{2}=\ln l_{2}^{-}-\left(\bar{a}_{2} \omega+G_{2}\right)<u_{2}(\eta) \leq u_{2}\left(\xi_{2}\right)<\ln l_{2}^{+} .
$$

On the other hand, in the light of the first equation of (25) and (26), we have

$$
\begin{aligned}
\bar{a}_{1} \omega+ & 2 g_{1} \\
& =\int_{0}^{\omega}\left\{b_{1}(t) e^{u_{1}\left(t-\tau_{11}(t)\right)}+h_{1}(t) e^{-u_{1}(t)}\right\} \mathrm{d} t \\
& \leq \bar{b}_{1} \omega e^{u_{1}\left(\xi_{1}\right)}+\bar{h}_{1} \omega e^{-u_{1}\left(\eta_{1}\right)} \\
& <\bar{b}_{1} \omega e^{u_{1}\left(\xi_{1}\right)}+\bar{h}_{1} \omega e^{-u_{1}\left(\xi_{1}\right)+(1 / 2)\left[\int_{0}^{\omega}\left|\dot{u}_{1}(s)\right| \mathrm{d} s+\sum_{k=1}^{q}\left|\Delta u_{1}\left(t_{k}\right)\right|\right]} \\
& <\bar{b}_{1} \omega e^{u_{1}\left(\xi_{1}\right)}+\bar{h}_{1} \omega e^{-u_{1}\left(\xi_{1}\right)+a_{1} \omega+G_{1}},
\end{aligned}
$$

namely,

$$
\bar{b}_{1} e^{2 u_{1}\left(\xi_{1}\right)}-\left(\bar{a}_{1}+\frac{2 g_{1}}{\omega}\right) e^{u_{1}\left(\xi_{1}\right)}+\bar{h}_{1} e^{\bar{a}_{1} \omega+G_{1}}>0,
$$

which implies that

$$
\begin{aligned}
& \ln A_{1}^{+}<u_{1}\left(\xi_{1}\right) \quad \text { or } \quad u_{1}\left(\xi_{1}\right)<\ln A_{1}^{-}, \\
\bar{a}_{1} \omega+ & 2 g_{1} \\
= & \int_{0}^{\omega}\left\{b_{1}(t) e^{u_{1}\left(t-\tau_{11}(t)\right)}+h_{1}(t) e^{-u_{1}(t)}\right\} \mathrm{d} t \\
\leq & \omega\left(\bar{b}_{1} e^{u_{1}\left(\xi_{1}\right)}+\bar{h}_{1} e^{-u_{1}\left(\eta_{1}\right)}\right) \\
< & \omega\left(\bar{b}_{1} e^{u_{1}\left(\eta_{1}\right)+(1 / 2)\left[\int_{0}^{\omega}\left|\dot{u}_{1}(s)\right| \mathrm{d} s+\sum_{k=1}^{q}\left|\Delta u_{1}\left(t_{k}\right)\right|\right]}+\bar{h}_{1} e^{-u_{1}\left(\eta_{1}\right)}\right) \\
< & \bar{b}_{1} \omega e^{u_{1}\left(\eta_{1}\right)+\bar{a}_{1} \omega+G_{1}}+\bar{h}_{1} \omega e^{-u_{1}\left(\eta_{1}\right)},
\end{aligned}
$$

that is,

$$
\bar{b}_{1} e^{2 u_{1}\left(\eta_{1}\right)}-\left(\bar{a}_{1}+\frac{2 g_{1}}{\omega}\right) e^{-\left(\bar{a}_{1} \omega+G_{1}\right)} e^{u_{1}\left(\eta_{1}\right)}+\bar{h}_{1} e^{-\left(\bar{a}_{1} \omega+G_{1}\right)}>0,
$$

which implies that

$$
\ln B_{1}^{+}<u_{1}\left(\eta_{1}\right) \quad \text { or } \quad u_{1}\left(\eta_{1}\right)<\ln B_{1}^{-} \text {. }
$$


By (25) and (31), we have

$$
\begin{aligned}
2 g_{2}< & \bar{a}_{2} \omega+2 g_{2} \\
= & \int_{0}^{\omega}\left\{b_{2}(t) e^{u_{2}\left(t-\tau_{22}(t)\right)}\right. \\
& \left.\quad-\lambda c(t) e^{u_{1}\left(t-\tau_{21}(t)\right)}+h_{2}(t) e^{-u_{2}(t)}\right\} \mathrm{d} t \\
& <\bar{b}_{2} \omega e^{u_{2}\left(\xi_{2}\right)}+\bar{h}_{2} \omega e^{-u_{2}\left(\eta_{2}\right)} \\
< & \bar{b}_{2} \omega e^{u_{2}\left(\xi_{2}\right)}+\bar{h}_{2} \omega e^{-u_{2}\left(\xi_{2}\right)+(1 / 2)\left[\int_{0}^{\omega}\left|\dot{u}_{2}(s)\right| \mathrm{d} s+\sum_{k=1}^{q}\left|\Delta u_{2}\left(t_{k}\right)\right|\right]} \\
< & \bar{b}_{2} \omega e^{u_{2}\left(\xi_{2}\right)}+\bar{h}_{2} \omega e^{-u_{2}\left(\xi_{2}\right)+\bar{a}_{2} \omega+G_{2}},
\end{aligned}
$$

namely,

$$
\bar{b}_{2} e^{2 u_{2}\left(\xi_{2}\right)}-\left(\bar{a}_{2}+\frac{2 g_{2}}{\omega}\right) e^{u_{2}\left(\xi_{2}\right)}+\bar{h}_{2} e^{\bar{a}_{2} \omega+G_{2}}>0,
$$

which implies that

$$
\begin{gathered}
\ln A_{2}^{+}<u_{2}\left(\xi_{2}\right) \quad \text { or } \quad u_{2}\left(\xi_{2}\right)<\ln A_{2}^{-}, \\
2 g_{2}<\bar{a}_{2} \omega+2 g_{2} \\
=\int_{0}^{\omega}\left\{b_{2}(t) e^{u_{2}\left(t-\tau_{22}(t)\right)}\right. \\
\left.\quad-\lambda c(t) e^{u_{1}\left(t-\tau_{21}(t)\right)}+h_{2}(t) e^{-u_{2}(t)}\right\} \mathrm{d} t \\
<\bar{b}_{2} \omega e^{u_{2}\left(\xi_{2}\right)}+\bar{h}_{2} \omega e^{-u_{2}\left(\eta_{2}\right)} \\
<\bar{b}_{2} \omega e^{u_{2}\left(\eta_{2}\right)+(1 / 2)\left[\int_{0}^{\omega}\left|\dot{u}_{2}(s)\right| \mathrm{d} s+\sum_{k=1}^{q}\left|\Delta u_{2}\left(t_{k}\right)\right|\right]}+\bar{h}_{2} \omega e^{-u_{2}\left(\eta_{2}\right)} \\
<\bar{b}_{2} \omega e^{u_{2}\left(\eta_{2}\right)+\bar{a}_{2} \omega+G_{2}}+\bar{h}_{2} \omega e^{-u_{2}\left(\eta_{2}\right)},
\end{gathered}
$$

that is,

$$
\bar{b}_{2} e^{2 u_{2}\left(\eta_{2}\right)}-\left(\frac{2 g_{2}}{\omega}+\bar{a}_{2}\right) e^{-\left(\bar{a}_{2} \omega+G_{2}\right)} e^{u_{2}\left(\eta_{2}\right)}+\bar{h}_{2} e^{-\left(\bar{a}_{2} \omega+G_{2}\right)}>0,
$$

which implies that

$$
\ln B_{2}^{+}<u_{2}\left(\eta_{2}\right) \quad \text { or } \quad u_{2}\left(\eta_{2}\right)<\ln B_{2}^{-} \text {. }
$$

In view of (30)-(45) and Lemma 6, we have

$$
\begin{aligned}
& \min \left\{\ln A_{1}^{+}, \ln B_{1}^{+}\right\}<u_{1}\left(\eta_{1}\right)<u_{1}\left(\xi_{1}\right)<\ln l_{1}^{+}, \\
& \min \left\{\ln A_{2}^{+}, \ln B_{2}^{+}\right\}<u_{2}\left(\eta_{2}\right)<u_{2}\left(\xi_{2}\right)<\ln l_{2}^{+}
\end{aligned}
$$

or

$$
\begin{aligned}
& \ln H_{1}<u_{1}\left(\eta_{1}\right)<u_{1}\left(\xi_{1}\right)<\max \left\{\ln A_{1}^{-}, \ln B_{1}^{-}\right\}, \\
& \ln H_{2}<u_{2}\left(\eta_{2}\right)<u_{2}\left(\xi_{2}\right)<\max \left\{\ln A_{2}^{-}, \ln B_{2}^{-}\right\},
\end{aligned}
$$

which imply that, for all $t \in R$,

$$
\begin{aligned}
& \min \left\{\ln A_{1}^{+}, \ln B_{1}^{+}\right\}<u_{1}(t)<\ln l_{1}^{+} \text {or } \\
& \ln H_{1}^{-}<u_{1}(t)<\max \left\{\ln A_{1}^{-}, \ln B_{1}^{-}\right\}, \\
& \min \left\{\ln A_{2}^{+}, \ln B_{2}^{+}\right\}<u_{2}(t)<\ln l_{2}^{+} \text {or } \\
& \ln H_{2}^{-}<u_{2}(t)<\max \left\{\ln A_{2}^{-}, \ln B_{2}^{-}\right\} .
\end{aligned}
$$

Clearly, $\ln l_{1}^{ \pm}, \ln l_{2}^{ \pm}, \ln A_{1}^{ \pm}, \ln A_{2}^{ \pm}, \ln B_{1}^{ \pm}$, and $\ln B_{2}^{ \pm}$are independent of $\lambda$. Now let

$$
\begin{gathered}
\Omega_{1}=\left\{u=\left(u_{1}, u_{2}\right)^{T} \in X:\right. \\
\min \left\{\ln A_{1}^{+}, \ln B_{1}^{+}\right\}<u_{1}(t)<\ln l_{1}^{+}, \\
\left.\min \left\{\ln A_{2}^{+}, \ln B_{2}^{+}\right\}<u_{2}(t)<\ln l_{2}^{+}\right\}, \\
\Omega_{2}=\left\{u=\left(u_{1}, u_{2}\right)^{T} \in X:\right. \\
\min \left\{\ln A_{1}^{+}, \ln B_{1}^{+}\right\}<u_{1}(t) \\
\left.\ln H_{2}<u_{2}(t)<\max \left\{\ln A_{2}^{-}, \ln B_{2}^{-}\right\}\right\}, \\
\Omega_{3}=\left\{u=\left(u_{1}, u_{2}\right)^{T} \in X:\right. \\
\ln H_{1}<u_{1}(t)<\max \left\{\ln A_{1}^{-}, \ln B_{1}^{-}\right\}, \\
\left.\min \left\{\ln A_{2}^{+}, \ln B_{2}^{+}\right\}<u_{2}(t)<\ln l_{2}^{+}\right\}, \\
\Omega_{4}=\left\{u=\left(u_{1}, u_{2}\right)^{T} \in X:\right. \\
\ln H_{1}<u_{1}(t)<\max \left\{\ln A_{1}^{-}, \ln B_{1}^{-}\right\}, \\
\left.\ln H_{2}<u_{2}(t)<\max \left\{\ln A_{2}^{-}, \ln B_{2}^{-}\right\}\right\} .
\end{gathered}
$$

Obviously, the number of the above sets is four. We denote these sets as $\Omega_{k}, k=1,2,3$, and $4 . \Omega_{k}$ are bounded open subsets of $X, \Omega_{i} \cap \Omega_{j}=\phi, \quad i \neq j$. Thus $\Omega_{k}(k=1,2,3$, and 4) satisfies the requirement (a) in Lemma 1 .

Now we show that (b) of Lemma 1 holds; that is, we prove when $u \in \partial \Omega_{k} \cap \operatorname{Ker} L=\partial \Omega_{k} \cap R^{2}, Q N(u, 0) \neq(0,0,)^{T}, k=$ $1,2,3$, and 4 . If it is not true, then when $u \in \partial \Omega_{k} \cap \operatorname{Ker} L=$ $\partial \Omega_{k} \cap R^{2}, i=1,2,3$, and 4 , constant vector $u=\left(u_{1}, u_{2}\right)^{T}$ with $u \in \partial \Omega_{k}(k=1,2,3$, and 4) satisfies

$$
\begin{gathered}
-2 g_{1}=\int_{0}^{\omega}\left\{a_{1}(t)-b_{1}(t) e^{u_{1}\left(t-\tau_{11}(t)\right)}-h_{1}(t) e^{-u_{1}(t)}\right\} \mathrm{d} t, \\
-2 g_{2}=\int_{0}^{\omega}\left\{a_{2}(t)-b_{2}(t) e^{u_{2}\left(t-\tau_{22}(t)\right)}\right. \\
\left.+\lambda c(t) e^{u_{1}\left(t-\tau_{21}(t)\right)}-h_{2}(t) e^{-u_{2}(t)}\right\} \mathrm{d} t,
\end{gathered}
$$

that is,

$$
\begin{aligned}
-\frac{2 g_{1}}{\omega} & =\bar{a}_{1}-\bar{b}_{1} e^{u_{1}}-\bar{h}_{1} e^{-u_{1},}, \\
-\frac{2 g_{2}}{\omega} & =\bar{a}_{2}-\bar{b}_{2} e^{u_{2}}-\bar{h}_{2} e^{-u_{2}} .
\end{aligned}
$$


Similar to the process of (25)-(48), we obtain

$$
\begin{aligned}
\ln H_{i} & <u_{i}^{-}<\max \left\{\ln A_{i}^{-}, \ln B_{i}^{-}\right\} \\
& <\min \left\{\ln A_{i}^{+}, \ln B_{i}^{+}\right\} \\
& <u_{i}^{+}<\ln l_{i}^{+} \quad(1 \leq i \leq 2) .
\end{aligned}
$$

Then $u$ belongs to one of $\Omega_{k} \cap R^{2}, k=1,2,3$, and 4. This contradicts the fact that $u \in \partial \Omega_{k} \cap R^{2}, k=1,2,3$, and 4. This proves that (b) in Lemma 1 holds.

Finally, in order to show that (c) in Lemma 1 holds, we only prove that for $u \in \partial \Omega_{k} \cap \operatorname{Ker} L=\partial \Omega_{k} \cap$ $R^{2}, k=1,2,3$, and 4 , then it holds that $\operatorname{deg}\left\{\operatorname{JQN}(u, 0), \Omega_{k} \cap\right.$ $\left.\operatorname{Ker} L,(0,0)^{T}\right\} \neq 0$. To this end, we define the mapping $\phi$ : $\operatorname{Dom} L \times[0,1] \rightarrow X$ by

$$
\phi(u, \mu)=\mu Q N(u, 0)+(1-\mu) G(u),
$$

here $\mu \in[0,1]$ is a parameter, and $G(u)$ is defined by $G(u)$

$$
\begin{gathered}
=\left(\left(\begin{array}{l}
\frac{1}{\omega} \int_{0}^{\omega}\left(a_{1}(s)-b_{1}(s) e^{u_{1}(s)}-h_{1}(s) e^{-u_{1}(s)}\right) \mathrm{d} s+\frac{2 g_{1}}{\omega} \\
\frac{1}{\omega} \int_{0}^{\omega}\left(a_{2}(s)-b_{2}(s) e^{u_{2}(s)}-h_{2}(s) e^{-u_{2}(s)}\right) \mathrm{d} s+\frac{2 g_{2}}{\omega}
\end{array}\right),\right. \\
\left.\left\{\left(\begin{array}{l}
0 \\
0
\end{array}\right)\right\}_{k=1}^{q}\right) .
\end{gathered}
$$

We show that for $u \in \partial \Omega_{k} \cap \operatorname{Ker} L=\partial \Omega_{k} \cap R^{2}, k=1,2,3$, and 4, $\mu \in[0,1]$, then it holds that $\phi(u, \mu) \neq(0,0,)^{T}$. Otherwise, parameter $\mu$ and constant vector $u=\left(u_{1}, u_{2}\right)^{T} \in R^{2}$ satisfy $\phi(u, \mu)=(0,0)^{T}$, that is,

$$
\begin{aligned}
0= & \mu \int_{0}^{\omega}\left(a_{1}(s)-b_{1}(s) e^{u_{1}}-h_{1}(s) e^{-u_{1}}+\frac{2 g_{1}}{\omega}\right) \mathrm{d} s \\
& +(1-\mu) \int_{0}^{\omega}\left(a_{1}(s)-b_{1}(s) e^{u_{1}}-h_{1}(s) e^{-u_{1}}+\frac{2 g_{1}}{\omega}\right) \mathrm{d} s, \\
0= & \mu \int_{0}^{\omega}\left(a_{2}(s)-b_{2}(s) e^{u_{2}}-h_{2}(s) e^{-u_{2}}+\frac{2 g_{2}}{\omega}\right) \mathrm{d} s \\
& +(1-\mu) \int_{0}^{\omega}\left(a_{2}(s)-b_{2}(s) e^{u_{2}}-h_{2}(s) e^{-u_{2}}+\frac{2 g_{2}}{\omega}\right) \mathrm{d} s,
\end{aligned}
$$

that is,

$$
\begin{aligned}
& \bar{a}_{1}-\bar{b}_{1} e^{u_{1}}-\bar{h}_{1} e^{-u_{1}}=-\frac{2 g_{1}}{\omega}, \\
& \bar{a}_{2}-\bar{b}_{2} e^{u_{2}}-\bar{h}_{2} e^{-u_{2}}=-\frac{2 g_{2}}{\omega} .
\end{aligned}
$$

Following the argument of (25)-(48), we obtain

$$
\begin{aligned}
\ln H_{i} & <u_{i}^{-}<\max \left\{\ln A_{i}^{-}, \ln B_{i}^{-}\right\} \\
& <\min \left\{\ln A_{i}^{+}, \ln B_{i}^{+}\right\} \\
& <u_{i}^{+}<\ln l_{i}^{+} \quad(1=1,2) .
\end{aligned}
$$

Equation (57) gives that $u$ belongs to one of $\Omega_{k} \cap R^{2}, k=$ $1,2,3$, and 4 . This contradicts the fact that $u \in \partial \Omega_{k} \cap R^{2}, \quad k=$ $1,2,3$, and 4 . This proves $\phi(u, \mu) \neq(0,0)^{T}$ holds. Note that the system of algebraic equations,

$$
\begin{aligned}
& \bar{a}_{1}-\bar{b}_{1} e^{x}-\bar{h}_{1} e^{-x}=-\frac{2 g_{1}}{\omega}, \\
& \bar{a}_{2}-\bar{b}_{2} e^{y}-\bar{h}_{2} e^{-y}=-\frac{2 g_{2}}{\omega},
\end{aligned}
$$

has four distinct solutions since $\left(T_{1}\right)$ and $\left(T_{2}\right)$ hold:

$$
\begin{aligned}
& \left(x_{1}^{*}, y_{1}^{*}\right)=\left(\ln x^{+}, \ln y^{+}\right), \\
& \left(x_{2}^{*}, y_{2}^{*}\right)=\left(\ln x^{+}, \ln y^{-}\right), \\
& \left(x_{3}^{*}, y_{3}^{*}\right)=\left(\ln x^{-}, \ln y^{+}\right), \\
& \left(x_{4}^{*}, y_{4}^{*}\right)=\left(\ln x^{-}, \ln y^{-}\right),
\end{aligned}
$$

where

$$
\begin{aligned}
& x^{ \pm}=\frac{\bar{a}_{1}+2 g_{1} / \omega \pm \sqrt{\left(\bar{a}_{1}+2 g_{1} / \omega\right)^{2}-4 \bar{b}_{1} \bar{h}_{1}}}{2 \bar{b}_{1}}, \\
& y^{ \pm}=\frac{\bar{a}_{2}+2 g_{2} / \omega \pm \sqrt{\left(\bar{a}_{2}+2 g_{2} / \omega\right)^{2}-4 \bar{b}_{2} \bar{h}_{2}}}{2 \bar{b}_{2}} .
\end{aligned}
$$

Similar to the proof of Lemma 6, it is easy to verify that

$$
\begin{aligned}
\ln H_{1}^{-} & <\ln x^{-}<\max \left\{\ln B_{1}^{-}, \ln A_{1}^{-}\right\} \\
& <\min \left\{\ln A_{1}^{+}, \ln B_{1}^{+}\right\}<\ln x^{+}<\ln l_{1}^{+}, \\
\ln H_{2}^{-} & <\ln y^{-}<\max \left\{\ln B_{2}^{-}, \ln A_{2}^{-}\right\} \\
& <\min \left\{\ln A_{2}^{+}, \ln B_{2}^{+}\right\}<\ln y^{+}<\ln l_{2}^{+} .
\end{aligned}
$$

Therefore

$$
\begin{array}{ll}
\left(x_{1}^{*}, y_{1}^{*}\right) \in \Omega_{1}, & \left(x_{2}^{*}, y_{2}^{*}\right) \in \Omega_{2}, \\
\left(x_{3}^{*}, y_{3}^{*}\right) \in \Omega_{3}, \quad\left(x_{4}^{*}, y_{4}^{*}\right) \in \Omega_{4},
\end{array}
$$

uniquely belongs to the corresponding $\Omega_{k}$. Since $\operatorname{Ker} L=$ $\operatorname{Im} \mathrm{Q}$, we can take $J=I$. A direct computation gives, for $k=1,2,3$, and 4 ,

$$
\begin{aligned}
\operatorname{deg}\left\{\operatorname{JQN}(u, 0), \Omega_{k} \cap \operatorname{Ker} L,(0,0)^{T}\right\} \\
\quad=\operatorname{sign}\left|\begin{array}{cc}
-\bar{b}_{1} x^{*}+\frac{\bar{h}_{1}}{x^{*}} & 0 \\
0 & -\bar{b}_{2} y^{*}+\frac{\bar{h}_{2}}{y^{*}}
\end{array}\right| \\
=\operatorname{sign}\left[\left(-\bar{b}_{1} x^{* 2}+\bar{h}_{1}\right)\left(-\bar{b}_{2} y^{* 2}+\bar{h}_{2}\right)\right] .
\end{aligned}
$$

Since $\left(\bar{a}_{1}+2 g_{1} / \omega\right)-\bar{b}_{1} x^{*}-\bar{h}_{1} / x^{*}=0,\left(\bar{a}_{2}+2 g_{2} / \omega\right)-\bar{b}_{2} y^{*}-$ $\bar{h}_{1} / y^{*}=0$. 
Hence,

$$
\begin{aligned}
& \operatorname{deg}\left\{\operatorname{JQN}(u, 0), \Omega_{1} \cap \operatorname{Ker} L,(0,0)^{T}\right\} \\
& =\operatorname{sign}\left\{\left(-\bar{b}_{1}\left(x_{1}^{*}\right)^{2}+\bar{h}_{1}\right)\left(-\bar{b}_{2}\left(y_{1}^{*}\right)^{2}+\bar{h}_{2}\right)\right\}=1, \\
& \operatorname{deg}\left\{\operatorname{JQN}(u, 0), \Omega_{2} \cap \operatorname{Ker} L,(0,0)^{T}\right\} \\
& \quad=\operatorname{sign}\left\{\left(-\bar{b}_{1}\left(x_{2}^{*}\right)^{2}+\bar{h}_{1}\right)\left(-\bar{b}_{2}\left(y_{2}^{*}\right)^{2}+\bar{h}_{2}\right)\right\}=-1, \\
& \operatorname{deg}\left\{\operatorname{JQN}(u, 0), \Omega_{3} \cap \operatorname{Ker} L,(0,0)^{T}\right\} \\
& \quad=\operatorname{sign}\left\{\left(-\bar{b}_{1}\left(x_{3}^{*}\right)^{2}+\bar{h}_{1}\right)\left(-\bar{b}_{2}\left(y_{3}^{*}\right)^{2}+\bar{h}_{2}\right)\right\}=1, \\
& \operatorname{deg}\left\{\operatorname{JQN}(u, 0), \Omega_{4} \cap \operatorname{Ker} L,(0,0)^{T}\right\} \\
& \quad=\operatorname{sign}\left\{\left(-\bar{b}_{1}\left(x_{4}^{*}\right)^{2}+\bar{h}_{1}\right)\left(-\bar{b}_{2}\left(y_{4}^{*}\right)^{2}+\bar{h}_{2}\right)\right\}=-1 .
\end{aligned}
$$

So far, we have proved that $\Omega_{k}(k=1,2,3$, and 4) satisfies all the assumptions in Lemma 1 . Hence, system (15) has at least four different $\omega$-periodic solutions. Thus by Lemma 1 , system (3) has at least four different positive $\omega$-periodic solutions. This completes the proof of Theorem 7.

\section{An Example}

Example 8. Consider the following time-delay parasitical system with impulsive effectiveness and harvesting terms:

$$
\begin{aligned}
& \dot{x}_{1}(t) \\
& =x_{1}(t)\left(\frac{3+\sin t}{2}-\frac{2+\sin t}{10 e^{2 \pi}} x_{1}(t-|\sin t|)\right)-\frac{4+\cos t}{20 e^{2 \pi}}, \\
& \dot{x}_{2}(t)=x_{2}(t) \\
& \times\left(\frac{2+\cos t}{25}-\frac{5+\cos t}{10 e^{\pi}} x_{2}(t-|\sin 2 t|)\right. \\
& \left.\quad+\frac{2+\sin 2 t}{8} x_{1}(t-|\cos 2 t|)\right)-\frac{2+\cos t}{25 e^{\pi}}, \\
& \Delta x_{1}\left(\frac{\pi}{2}\right)=e^{-\pi}-1, \quad \Delta x_{1}\left(\frac{3 \pi}{2}\right)=e^{\pi}-1, \\
& \Delta x_{2}\left(\frac{\pi}{2}\right)=e^{-\frac{\pi}{2}-1, \quad \Delta x_{2}\left(\frac{3 \pi}{2}\right)=e^{3 \pi / 2}-1 .}
\end{aligned}
$$

In this case, $a_{1}(t)=(3+\sin t) / 2, \quad b_{1}(t)=(2+\sin t) / 10 e^{2} \pi$, $h_{1}(t)=(4+\cos t) / 20 e^{2} \pi, \tau_{11}(t)=|\sin t|, a_{2}(t)=(2+\cos t) /$ $25, b_{2}(t)=(5+\cos t) / 10, h_{2}(t)=(2+\cos t) / 25 e^{\pi}, c(t)=$ $(2+\sin 2 t) / 8, \tau_{22}(t)=|\cos 2 t|$, and $\tau_{21}(t)=|\sin 2 t|$. It is not difficult to calculate $\rho_{1}>e^{4 \pi}>1, \rho_{2}=e^{2 \pi}>1$. Since

$$
\begin{aligned}
l_{1}^{ \pm} & =\frac{\left(\bar{a}_{1}+2 g_{1} / \omega\right) \rho_{1} \pm \sqrt{\left[\left(\bar{a}_{1}+2 g_{1} / \omega\right) \rho_{1}\right]^{2}-4 \bar{b}_{1} \bar{h}_{1} \rho_{1}}}{2 \bar{b}_{1}} \\
& =\frac{\left(15 e^{4 \pi} \pm 3 \sqrt{225 e^{8 \pi}-16}\right)}{4 e^{-2 \pi}},
\end{aligned}
$$

$$
\begin{aligned}
l_{2}^{ \pm}= & \left(\left(\bar{c} l_{1}^{+}+\bar{a}_{2}+2 g_{2} / \omega\right) \rho_{2}\right. \\
& \left. \pm \sqrt{\left[\left(\bar{c} l_{1}^{+}+\bar{a}_{2}+2 g_{2} / \omega\right) \rho_{2}\right]^{2}-4 \bar{b}_{2} \bar{h}_{2} \rho_{2}}\right)\left(2 \bar{b}_{2}\right)^{-1} \\
= & \frac{15 e^{2 \pi} \pm \sqrt{225 e^{4 \pi}-64}}{20 e^{-\pi}} .
\end{aligned}
$$

Then

$$
\begin{aligned}
& \frac{\bar{a}_{1}+2 g_{1} / \omega}{\sqrt{\rho_{1}}}=\frac{1}{2} e^{-2 \pi}>\frac{2}{5} e^{-2 \pi}=2 \sqrt{\bar{b}_{1} \bar{h}_{1}}, \\
& \frac{2 g_{2} / \omega+\bar{a}_{2}}{\sqrt{\rho_{2}}}=\frac{21}{25} e^{-\pi}>\frac{2}{5} e^{-\pi}=2 \sqrt{\bar{b}_{2} \bar{h}_{2}} .
\end{aligned}
$$

Therefore, all conditions of Theorem 7 are satisfied. By Theorem 7 , system (65) has at least four positive $2 \pi$-periodic solutions.

\section{Aknowledgment}

This work is supported by Yunnan Province Education Department Scientific Research Fund Project (no. 2012Z065).

\section{References}

[1] Z. Ma, Mathematical Modelling and Studing on Species Ecology, Education Press, Hefei, China, 1996.

[2] H. R. Thieme, Mathematics in Population Biology, Princeton Series in Theoretical and Computational Biology, Princeton University Press, Princeton, NJ, USA, 2003.

[3] K. Zhao and Y. Ye, "Four positive periodic solutions to a periodic Lotka-Volterra predatory-prey system with harvesting terms," Nonlinear Analysis: Real World Applications, vol. 11, no. 4, pp. 2448-2455, 2010.

[4] D. Baĭnov and P. Simeonov, Impulsive Differential Equations: Periodic Solutions and Applications, vol. 66 of Pitman Monographs and Surveys in Pure and Applied Mathematics, Longman Scientific \& Technical, Harlow, UK, 1993.

[5] V. Lakshmikantham, D. D. Baĭnov, and P. S. Simeonov, Theory of Impulsive Differential Equations, vol. 6 of Series in Modern Applied Mathematics, World Scientific Publishing, Teaneck, NJ, USA, 1989.

[6] Y. Chen, "Multiple periodic solutions of delayed predator-prey systems with type IV functional responses," Nonlinear Analysis. Real World Applications, vol. 5, no. 1, pp. 45-53, 2004.

[7] Q. Wang, B. Dai, and Y. Chen, "Multiple periodic solutions of an impulsive predator-prey model with Holling-type IV functional response," Mathematical and Computer Modelling, vol. 49, no. 910, pp. 1829-1836, 2009.

[8] D. Hu and Z. Zhang, "Four positive periodic solutions to a Lotka-Volterra cooperative system with harvesting terms," Nonlinear Analysis. Real World Applications, vol. 11, no. 2, pp. 1115-1121, 2010.

[9] R. E. Gaines and J. L. Mawhin, Coincidence Degree, and Nonlinear Differential Equations, vol. 568 of Lecture Notes in Mathematics, Springer, Berlin, Germany, 1977. 


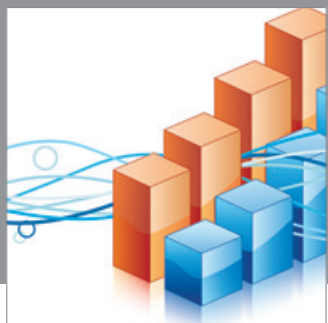

Advances in

Operations Research

mansans

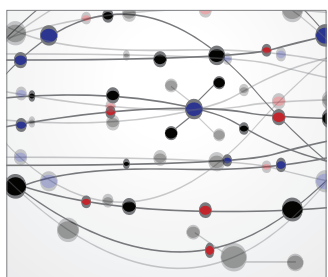

The Scientific World Journal
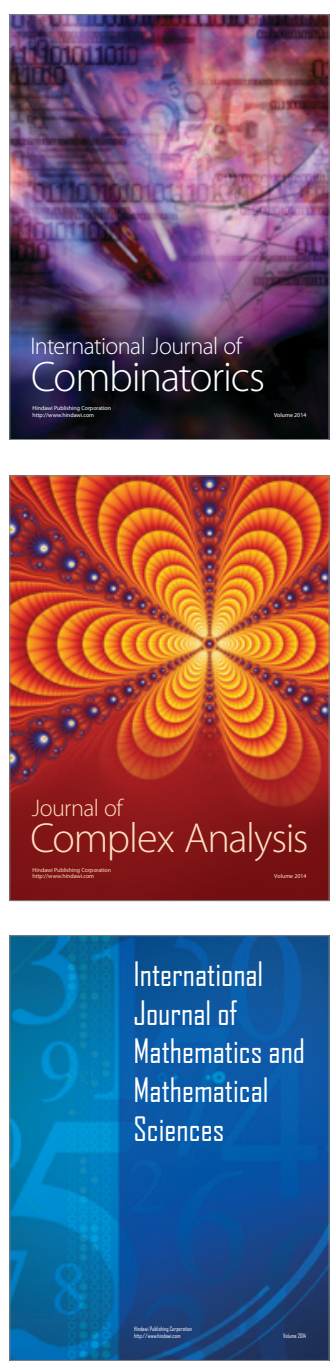
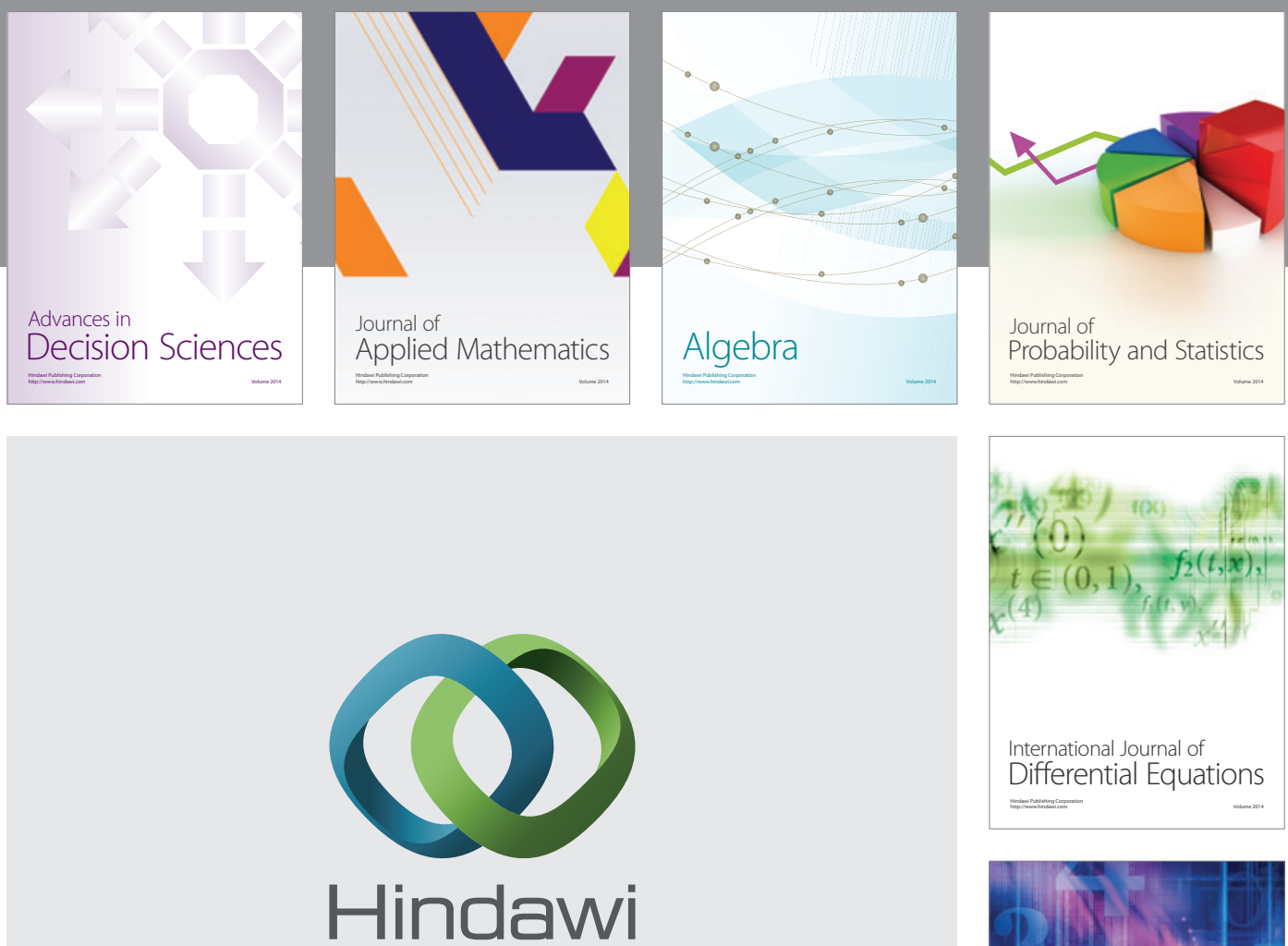

Submit your manuscripts at http://www.hindawi.com
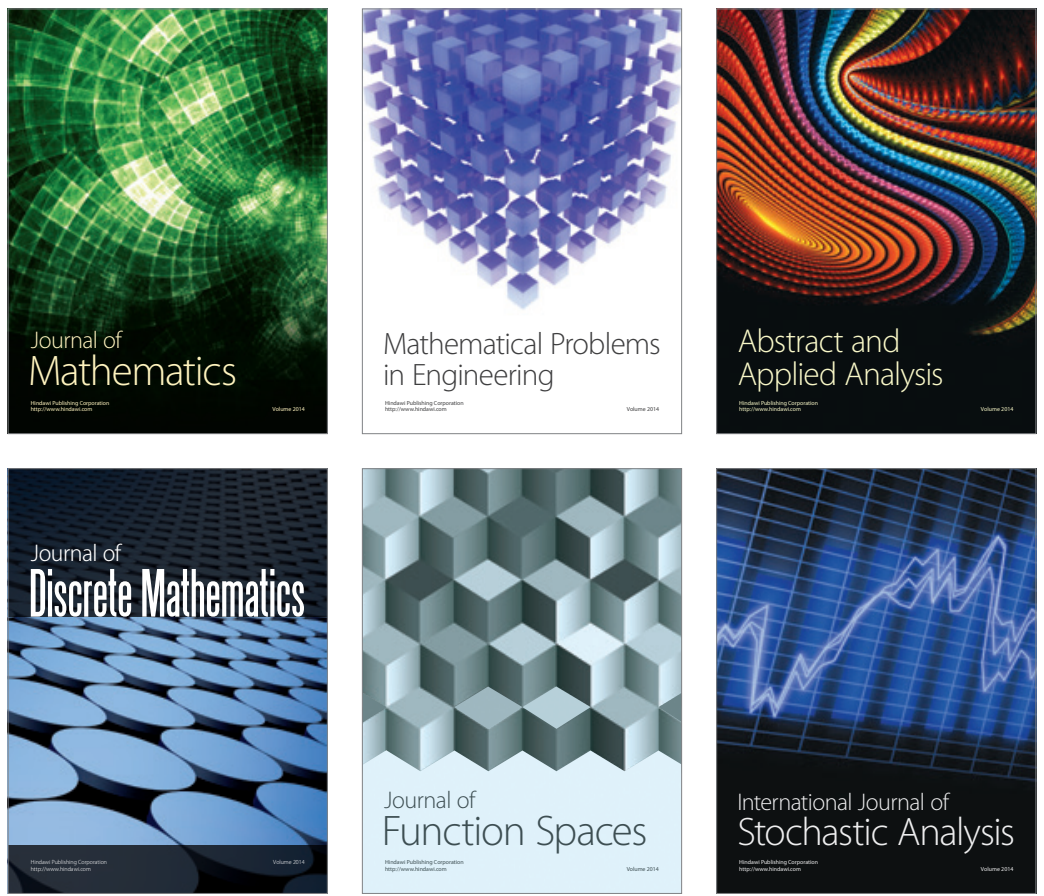

Journal of

Function Spaces

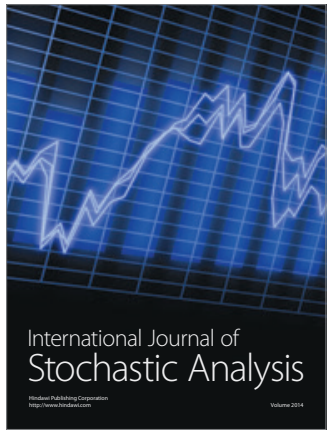

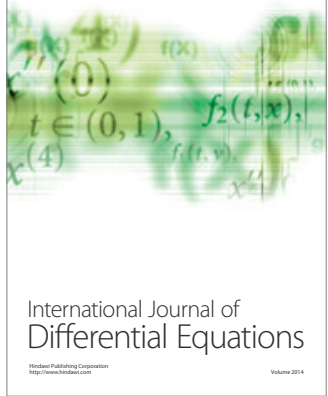
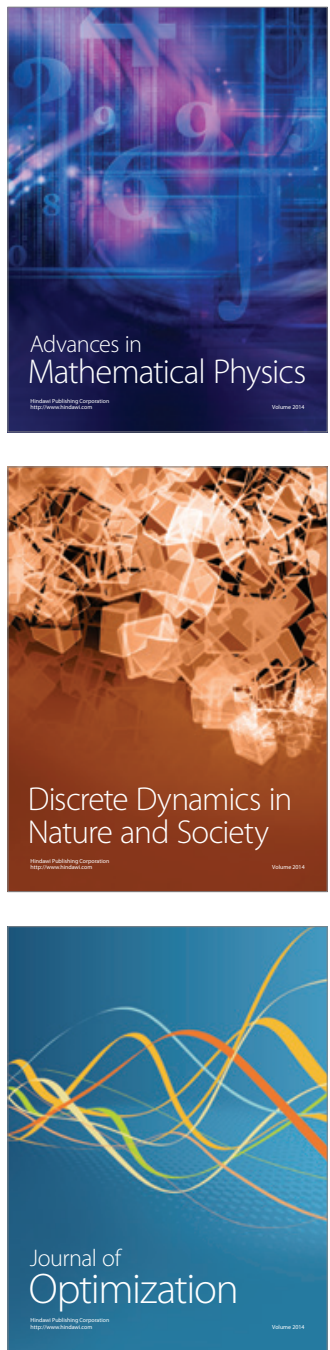